\title{
Comportamento magnético de materiais por meio da mecânica estatística
}

\author{
Magnetic behavior of materials through statistical mechanics \\ L. M. Holanda*回, I. R. O. Ramos ${ }^{2}$, A. P. Lima ${ }^{3}$, J. P. M. Braga ${ }^{4}$, H. T. C. M. Souza ${ }^{1}$ \\ ${ }^{1}$ Universidade Federal Rural do Semi-Árido, Campus Pau dos Ferros, Pau dos Ferros, RN, Brasil \\ ${ }^{2}$ Universidade Estadual Vale do Acaraú, Campus Cidao, Sobral, CE, Brasil \\ ${ }^{3}$ Universidade Federal do Rio Grande do Norte, Escola de Ciência e Tecnologia, Natal, RN, Brasil \\ ${ }^{4}$ Universidade da Integração Internacional da Lusofonia Afro-Brasileira, Campus dos Palmares, Acarape, CE, Brasil
}

\begin{abstract}
Recebido em 06 de Agosto de 2019. Revisado em 09 de Outubro de 2019. Aceito em 16 de Outubro de 2019
Um problema importante no estudo de materiais magnéticos é saber como esses sistemas se comportam na presença de um campo magnético externo. A resposta para esse problema está na susceptibilidade magnética. Neste trabalho realizamos uma abordagem estatística das propriedades magnéticas dos materiais levando em consideração suas interações microscópicas. Nosso intuito é apresentar de maneira didática e acessível para um estudante de graduação as origens do fenômeno magnético e suas fases termodinâmicas, paramagnética, ferromagnética e antiferromagnética, calculando suas susceptibilidades e analisando seu comportamento em função da temperatura. Palavras-chave: Eletromagnetismo, Materiais Magnéticos, Susceptibilidade Magnética.
\end{abstract}

An important problem in studying magnetic materials is to know how these systems behave in the presence of an external magnetic field. The answer to that problem is in the magnetic susceptibility. In this work, we performed a statistical approach of the magnetic properties of materials that take into account their microscopic interactions. Our intent is to present in a didactic and accessible way to an undergraduate student the origins of the magnetic phenomenon and its thermodynamic, paramagnetic, ferromagnetic and antiferromagnetic phases, calculating its susceptibilities and analyzing its behavior as a function of temperature.

Keywords: Electromagnetism, Magnetic Materials, Magnetic Susceptibility.

\section{Introdução}

O estudo do eletromagnetismo de uma maneira mais contextualizada a partir de uma visão histórica ou através de suas várias aplicações, juntamente com exemplos didáticos, tem sido amplamente utilizado nos últimos anos e vem contribuindo de forma positiva para a melhoria do ensino de graduação nos cursos de Física. Em um trabalho anterior [1], abordamos dois problemas relacionando a Teoria do Eletromagnetismo (TEM) à Teoria da Relatividade Especial (TRE). Tais problemas se mostraram de fundamental importância para uma completa compreensão da natureza dos campos elétrico e magnético, bem como para o desenvolvimento dos conceitos da TRE. Em um segundo trabalho [2], com intuito de propiciar aos estudantes aplicações dos conteúdos estudados em cursos de Eletromagnetismo, mostramos como devem ser adaptadas as principais equações da eletrostática para o estudo de colóides carregados. Seguindo na mesma linha, neste trabalho pretendemos aprofundar a discussão sobre as propriedades magnéticas dos materiais através de um tratamento estatístico, levando em consideração suas interações microscópicas para deduzir seus compor-

*Endereço de correspondência: lino.holanda@ufersa.edu.br tamentos magnéticos dependentes da temperatura. Nesse sentido, este trabalho é complementar e mais detalhado que aquele apresentado por Ribeiro [3] no sentido de elucidar os detalhes das interações e classificá-las de acordo com suas especificidades.

As primeiras evidências relacionadas aos fenômenos magnéticos são atribuídas aos antigos gregos. Seu nome deriva da cidade de Magnésia, uma antiga cidade da província da Ásia Menor, onde foram registradas as primeiras observações 3 5]. Suas observações foram bem rudimentares, apenas pequenas atrações ou repulsões entre pequenos pedaços de um óxido de ferro, hoje conhecido como magnetita $\left(\mathrm{Fe}_{3} \mathrm{O}_{4}\right)$. Apesar de os mecanismos responsáveis por tais propriedades ainda fossem desconhecidos, havia muita especulação pelas diversas correntes de pensamento filosófico. O próprio Tales de Mileto afirmava que a substância tinha "alma"e podia atrair pedaços de matéria inanimada, "aspirando-os". Segundo ele, as substâncias tinham vontades e desejos como se fossem seres vivos 3 .

Mesmo gregos e romanos já conhecendo essa força misteriosa e os chineses usando-a na navegação, o magnetismo só começou a ser estudado com rigor pelo inglês William Gilbert (1544-1603), que chamava a força mag- 
nética de "alma"da Terra e enfatizava a importância da experiência e do experimento, no entanto, não considerava a possibilidade de uma teoria matemática [6].

A primeira aplicação do magnetismo foi a bússola, utilizada principalmente como dispositivo de orientação no serviço naval. Os dados sobre sua descoberta ainda não são consensuais, embora muitos pesquisadores admitam que a bússola tenha sido inventada pelos chineses por volta de 1100 a.C. 3].

Avanços provenientes do magnetismo (e das propriedades magnéticas dos materiais) nos últimos dois séculos foram rápidos e as maiores conquistas surgiram da conexão do magnetismo com outras ciências. Aqui, destacamos a conexão entre magnetismo e eletricidade, os quais estão intrinsicamente ligados através das quatro equações de Maxwell que, ao serem desacopladas, dão origem a duas equações de onda para os campos elétrico e magnético. Essas ondas se propagam exatamente com a velocidade da luz, mostrando que a luz é, na verdade, uma onda eletromagnética. Essa demonstração reduziu a ótica a um ramo do eletromagnetismo e propiciou diversos avanços tecnológicos $4,7,8$.

São muitos os materiais utilizados na fabricação de aparelhos eletrônicos os quais exploram diretamente as propriedades magnéticas da matéria. Dentre eles, destacamse os compostos de Terras Raras (série dos Lantanídeos) pelo seu magnetismo presente em quase todos os elementos e, em alguns casos, pelo forte campo magnético apresentado (como o Neodímio). O magnetismo desses materiais origina-se em sua estrutura atômica, onde a camada $4 f^{n}$ incompleta $(0 \leq n \leq 14)$ proporciona o surgimento de ímãs microscópicos, conhecidos como momentos magnéticos, provenientes dos spins dos elétrons desemparelhados que, ao se acoplarem, dão origem ao magnetismo observado $5,9,11$.

O acoplamento entre spins atômicos resulta em diversas e interessantes propriedades magnéticas que podem aumentar o poder magnético do material, tornando-o um ímã com campo de grande intensidade ou mesmo destruí-lo completamente. De acordo com o tipo de acoplamento magnético as principais propriedades apresentadas pelos materiais são: diamagnetismo, paramagnetismo, ferromagnetismo (FM) e antiferromagnetismo (AFM) 4, 5, 12, 13.

O magnetismo tem influência direta na vida humana e suas aplicações parecem ilimitadas, indo desde instrumentos de armazenamento de dados e informações (devido à propriedade de histerese magnética presente em uma classe particular de materiais ferromagnéticos) até terapias de câncer com o auxílio de nanopartículas magnéticas.

Nas próximas seções, discutiremos de uma maneira didática as origens físicas microscópicas do magnetismo na matéria e calcularemos por meio de uma abordagem estatística as susceptibilidades de vários tipos de materiais magnéticos. Nossa proposta é apresentar esse assunto de maneira acessível a um estudante de graduação, de tal modo que o presente trabalho possa ser utilizado como material complementar em disciplinas de física estatística e eletromagnetismo.

\section{A origem do magnetismo dos materiais}

O magnetismo e, consequentemente, as propriedades magnéticas dos materiais, são fenômenos que têm origem na escala atômica. Para entender melhor essa definição, podemos pensar no átomo mais simples que existe (o átomo de Hidrogênio) e estender o tratamento para átomos com mais de um elétron. O movimento do elétron carregado em torno do núcleo pode ser caracterizado como uma pequena corrente elétrica e, portanto, teremos um momento magnético associado a esse movimento, o qual dará origem à grandeza fundamental do magnetismo, o momento de dipolo magnético $\vec{\mu}_{L}$ (momento de dipolo magnético orbital) [4,5]. Esse momento de dipolo pode ser pensado como um pequeno ímã em escala atômica gerando um pequeno campo magnético. Isso não é nada mais do que uma consequência da lei de Ampère-Maxwell de que fontes geradoras de campos magnéticos $\vec{B}$ são densidades de corrente elétrica $\overrightarrow{\mathcal{J}}$ ou campos elétricos $\vec{E}$ variáveis no tempo 77,8]. Escrita na forma diferencial, a lei de Ampère-Maxwell tem a forma

$$
\nabla \times \vec{B}=\mu_{0} \overrightarrow{\mathcal{J}}+\mu_{0} \epsilon_{0} \frac{\partial \vec{E}}{\partial t}
$$

em que $\mu_{0}$ e $\epsilon_{0}$ são, respectivamente, as constantes de permeabilidade magnética e permissividade elétrica do vácuo. O elétron, por sua vez, também tem um momento magnético intrínseco denominado spin $\vec{S}$, uma grandeza cuja origem é proveniente da Mecânica Quântica e não será tratada em detalhes aqui. Adotaremos simplesmente que o elétron possui um momento magnético intrínseco, denominado spin, e será representado por $\vec{\mu}_{S}$ 4, 5. Portanto, existem duas contribuições para o momento angular eletrônico, uma contribuição orbital e outra de spin.

Podemos associar o termo orbital a um circuito de uma única espira formada pelo elétron girando em torno do núcleo atômico. Esse elétron terá um momento magnético associado dado por $\vec{\mu}_{L}=I A \hat{n}$, onde $I$ representa a corrente elétrica, $A$ é a área da espira e $\hat{n}$ é o versor perpendicular à área da órbita do elétron [14,15. Se o raio da órbita for $r$ e o elétron gira em torno do núcleo atômico com velocidade angular $\omega$ e período $T$, então

$$
\mu_{L}=\frac{-e}{T} \pi r^{2}=\frac{-e \omega r^{2}}{2}
$$

A magnitude do momento angular $\vec{L}$ desse elétron, dado por $\vec{L}=\vec{r} \times \vec{p}=m_{e} \vec{r} \times \vec{v}$, fornece

$$
L=m_{e} \omega r^{2}
$$


em que $m_{e}$ é a massa do elétron. Portanto, a magnitude no momento magnético orbital é encontrada pela relação

$$
\mu_{L}=\frac{-e \omega r^{2}}{2}=\left(m_{e} \omega r^{2}\right) \frac{-e}{2 m_{e}}=\frac{-e}{2 m_{e}} L
$$

e representada vetorialmente por

$$
\vec{\mu}_{L}=\frac{-e}{2 m_{e}} \vec{L}
$$

Como a componente orbital do momento angular do elétron em uma direção arbitrária é quantizada em valores de $n \hbar(n=1,2,3, \ldots)$ [4, 16, 17], o menor valor de $L$ associado à direção $z$, por exemplo, é $L_{z}=\hbar$. Com base nesse resultado, é definido o menor valor do módulo de $\vec{\mu}_{L}$ como o magneton de Bohr

$$
\mu_{B}=\frac{e \hbar}{2 m_{e}}
$$

A componente de spin tem uma expressão semelhante, porém, com módulo duas vezes maior

$$
\vec{\mu}_{S}=\frac{-e}{m_{e}} \vec{S}
$$

O momento angular total, $\vec{J}$, será a soma dos momentos orbital $(\vec{L})$ e de $\operatorname{spin}(\vec{S})$

$$
\vec{J}=\vec{L}+\vec{S}
$$

Ao aplicarmos um campo magnético, $\vec{H}$, em um material que possui momento magnético, $\vec{\mu}$, a teoria eletromagnética clássica nos diz que esse momento magnético sofrerá um torque devido à presença do campo $7,14,15$, , dado por

$$
\vec{\tau}=\vec{\mu} \times \vec{H}=\frac{d \vec{J}}{d t}
$$

que tenderá a alinhá-lo na direção do campo aplicado. É natural, então, que a relação de proporcionalidade medida pela razão giromagnética, $\gamma_{g}=\frac{g \mu_{B}}{\hbar}$, entre o momento magnético e o momento angular, $\vec{\mu}=\gamma_{g} \vec{J}$, forneça a taxa de variação temporal de $\vec{\mu}$, escrita como

$$
\frac{d \vec{\mu}}{d t}=\gamma_{g} \vec{\mu} \times \vec{H}
$$

Se considerarmos, sem perda de generalidade, que o campo magnético está na direção $z$, as equações para o movimento de $\vec{\mu}$ nas três coordenadas ficam

$$
\begin{gathered}
\frac{d \mu_{x}}{d t}=\gamma_{g} \mu_{y} H=\omega_{0} \mu_{y} \\
\frac{d \mu_{y}}{d t}=-\gamma_{g} \mu_{x} H=-\omega_{0} \mu_{x} \\
\frac{d \mu_{z}}{d t}=0
\end{gathered}
$$

que são equações acopladas e podem ser resolvidas facilmente para encontrarmos

$$
\begin{gathered}
\mu_{x}=\left(\frac{g \mu_{B}}{2}\right) \operatorname{sen} \theta \cos \left(\omega_{0} t\right) \\
\mu_{y}=\left(\frac{g \mu_{B}}{2}\right) \operatorname{sen} \theta \operatorname{sen}\left(\omega_{0} t\right) \\
\mu_{z}=\left(\frac{g \mu_{B}}{2}\right) \cos \theta
\end{gathered}
$$

Observando a estrutura dessas equações, podemos fazer uma analogia com um vetor, no espaço tridimensional, girando em torno do campo magnético aplicado com uma frequência dada por $\omega_{0}=\gamma_{g} H$.

\section{Algumas propriedades dos materiais magnéticos}

Dada a definição de momento magnético, é possível compreender como as interações na matéria se manifestam em fenômenos associados ao magnetismo. Para isso, é interessante apresentar algumas propriedades magnéticas dos materiais e como elas se comportam na presença de um campo magnético aplicado, com uma análise dos conceitos e o formalismo matemático empregado na descrição desses fenômenos e suas propriedades.

\subsection{Paramagnetismo}

É a propriedade que certos materiais apresenta quando submetidos a um campo magnético externo de alinhar seus momentos magnéticos, na ausência de interações magnéticas, na direção do campo magnético aplicado. $\mathrm{O}$ paramagnetismo contribui positivamente para a susceptibilidade magnética. É observado em átomos, moléculas ou defeitos na rede cristalina contendo um número ímpar de elétrons (por exemplo, sódio, óxido nítrico gasoso (NO) e radicais orgânicos livres), em elementos de transição, terras raras e actinídeos (por exemplo, $\mathrm{Mn}^{2+}, \mathrm{Gd}^{3+}$, $\mathrm{U}^{4+}$ ) e em alguns metais [12].

Existem dois mecanismos possíveis para a contribuição paramagnética de uma substância, a saber: um devido aos elétrons desemparelhados e localizados em sítios de uma rede cristalina, conhecido como paramagnetismo de Curie, e outro devido aos elétrons de condução, no caso de metais, conhecido como paramagnetismo de Pauli. Vejamos em detalhe esses dois tipos de paramagnetismo.

\section{- Paramagnetismo de Curie}

É a contribuição paramagnética de átomos com elétrons desemparelhados, muito comum em íons de Terras Raras, onde os elétrons das camadas $4 f$, que são mais localizadas, dão origem ao paramagnetismo desses elementos.

Assumiremos um sistema com $\mathrm{N}$ átomos magnéticos idênticos com momento angular total $\vec{J}$ e momento magnético $\vec{\mu}_{J}$. Se os átomos não interagem entre si, a contribuição para o hamiltoniano vem de sua interação com um campo magnético aplicado e, como são idênticas, precisamos somente do hamiltoniano para um único átomo 18

$$
\mathcal{H}=-\vec{\mu}_{J} \cdot \vec{H}
$$

Podemos escolher, sem perda de generalidade, $\vec{H}$ ao longo do eixo $z$, tal que

$$
\mathcal{H}=-\mu_{J_{z}} H
$$

com $J_{z}=-J,-J+1, \ldots,+J$ e autovalores de energia dados por

$$
E_{m}=-m g \mu_{B} H
$$

A função de partição canônica $19 \sqrt{21}]$ será, portanto

$$
Z=\sum_{m=-J}^{J} e^{-\beta E_{m}}=\sum_{m=-J}^{J} e^{m g \mu_{B} H / k_{B} T}
$$


onde $\beta=1 / k_{B} T$ e $k_{B}=1,38064852 \times 10^{-23} \mathrm{~m}^{2} \cdot \mathrm{kg} \cdot \mathrm{s}^{-2}$ $K^{-1}$ é a constante de Boltzmann. Com isso, podemos encontrar a magnetização associada ao sistema de spins

$$
\begin{aligned}
M=N\left\langle\mu_{J}\right\rangle & =N g \mu_{B}\left\langle J_{z}\right\rangle \\
& =N g \mu_{B} \frac{\sum_{m=-J}^{J} m e^{m g \mu_{B} H / k_{B} T}}{\sum_{m=-J}^{J} e^{m g \mu_{B} H / k_{B} T}}
\end{aligned}
$$

que em termos de $Z$ pode ser escrita como

$$
M=N g \mu_{B} \frac{d}{d \gamma}(\ln Z)
$$

com $\gamma=\beta g \mu_{B} H$. Portanto, em termos de $\gamma$ a equação (14) fica

$Z=\sum_{m=-J}^{J} e^{m \gamma}=e^{-\gamma J}+e^{\gamma(-J+1)}+e^{\gamma(-J+2)}+\cdots+e^{\gamma J}$

Isolando $e^{-\gamma J}$, temos

$$
Z=e^{-\gamma J}\left(1+e^{\gamma}+e^{2 \gamma}+\cdots+e^{2 J \gamma}\right)
$$

Vemos que essa soma é uma progressão geométrica (P.G) de $2 \mathrm{~J}+1$ termos, cuja soma é dada por

$$
S_{n}=\frac{a_{1}\left(q^{n}-1\right)}{q-1}
$$

com $a_{1}=1$ e $q=e^{\gamma}$. Substituindo esses termos na equação (19), a soma da equação (18) torna-se

$$
Z=e^{-\gamma J}\left(\frac{e^{\gamma(2 J+1)}-1}{e^{\gamma}-1}\right)=\frac{e^{\gamma J} e^{\gamma}-e^{-\gamma J}}{e^{\gamma}-1}
$$

Multiplicando o numerador e o denominador desse resultado por $e^{-\gamma / 2}$, após algumas manipulações é possível reescrevê-lo na forma

$$
Z=\frac{e^{\gamma(J+1 / 2)}-e^{-\gamma(J+1 / 2)}}{e^{\gamma / 2}-e^{-\gamma / 2}}
$$

que nos fornece uma forma transcendental mais elegante da função de partição, dada por

$$
Z=\frac{\operatorname{senh}[\gamma(J+1 / 2)]}{\operatorname{senh}(\gamma / 2)}
$$

De acordo com a equação (16), para encontrarmos a magnetização do sistema, precisamos tomar o logaritmo de $Z$ e derivar a expressão resultante em relação à $\gamma$, ou seja,

$M=N g \mu_{B}\left\{(J+1 / 2) \operatorname{cotgh}[\gamma(J+1 / 2)]-\frac{1}{2} \operatorname{cotgh}(\gamma / 2)\right\}$ (23)
Substituindo o valor de $\gamma$ e fazendo $x=g \mu_{B} J H / k_{B} T$, a magnetização pode ser escrita como

$$
M=N g \mu_{B} J B_{J}(x)
$$

onde a função $B_{J}(x)$ é identificada como a função de Brillouin 22] e tem sua expressão dada por

$$
B_{J}(x)=\frac{2 J+1}{2 J} \operatorname{cotgh}\left(\frac{2 J+1}{2 J} x\right)-\frac{1}{2 J} \operatorname{cotgh}\left(\frac{x}{2 J}\right)
$$

Faremos inicialmente uma simplificação na expressão da magnetização para um caso particular comumente estudado e de grande importância para análises magnéticas: o caso de altas temperaturas, onde a energia térmica é muito maior que a energia magnética $\left(k_{B} T>>g \mu_{B} H\right.$ ou $x<<1$ ). Para obtermos uma expressão para este caso, precisamos expandir a função de Brillouin em série de Taylor até primeira ordem. Isso pode ser feito facilmente utilizando a expansão da cotangente hiperbólica em termos da função exponencial, ou seja,

$$
\begin{gathered}
\operatorname{cotgh}(x)=\frac{\operatorname{senh}(x)}{\cosh (x)}=\frac{e^{x}-e^{-x}}{e^{x}+e^{-x}} \\
\operatorname{cotgh}(x) \cong \frac{1}{x} \frac{\left(1+\frac{x^{2}}{2}\right)}{\left(1+\frac{x^{2}}{6}\right)} \quad x<<1
\end{gathered}
$$

Com isso, a expansão da cotangente hiperbólica fica

$$
\operatorname{cotgh}(x) \approx \frac{1}{x}+\frac{x}{3}
$$

e a equação (25) pode ser escrita como

$$
B_{J}(x)=\frac{J+1}{3 J} x
$$

Com essa aproximação, a magnetização para o caso de altas temperaturas fica

$$
M=\frac{N g^{2} \mu_{B}^{2} J(J+1)}{3 k_{B} T} H
$$

Assim, podemos encontrar a susceptibilidade magnética, $\chi$, de um paramagneto de momento angular total $\mathrm{J}[3-5$ 12,13

$$
\chi=\frac{M}{H}=\frac{N g^{2} \mu_{B}^{2} J(J+1)}{3 k_{B} T}
$$

Podemos definir $\mathrm{C}=N g^{2} \mu_{B}^{2} J(J+1) / 3 k_{B}$ como sendo a constante de Curie e, portanto, a susceptibilidade fica

$$
\chi=\frac{C}{T}
$$

Essa equação determina o comportamento magnético de um material paramagnético no regime específico de energia analisado. Aqui, vemos que seu poder magnético aumenta com o inverso da temperatura, ou seja, quando diminuimos $T$, o magnetismo dessa classe de materiais torna-se cada vez mais intenso, o que está em perfeito 
acordo com as observações.

O segundo caso, aplica-se quando a energia térmica é muito menor que a energia magnética $\left(k_{B} T<<g \mu_{B} H\right.$ ou $x>>1)$. Para este caso, tomaremos a expansão da cotangente hiperbólica para valores grandes de $x$, o que a reduz simplesmente a $\operatorname{cotgh}(x) \cong 1$. Portanto, a magnetização reduz-se a

$$
M=g \mu_{B} N J
$$

Neste caso os momentos magnéticos estão perfeitamente ordenados na direção do campo aplicado e, portanto, a magnetização atinge seu valor máximo, denominado magnetização de saturação, $M_{S}$.

É importante notar que a susceptibilidade magnética dada pela equação (31) é avaliada no limite de campo magnético muito fraco, a qual é descrita em termos do momento efetivo $\mu_{e f f}=g \mu_{B} \sqrt{(J(J+1)}$. No entanto, em altos campos, a magnetização satura em $M_{S}$ que, de acordo com a equação (33), é equivalente a um momento $g \mu_{B} J$ por íon. Estes dois valores são diferentes, exceto no limite clássico $J \rightarrow \infty$ 4].

Uma vez que o calor específico c está relacionado com a entropia do sistema S através da energia livre de Helmholtz, $F=-k_{B} T \ln Z$, mediante a relação $c=T(d S / d T)$, onde $S=-(\partial F / \partial T)_{H}$, é fácil mostrar que

$$
\begin{aligned}
c & =-\frac{g}{\mu_{B} H T}\left\{\gamma\left(J+\frac{1}{2}\right)^{2} \operatorname{cossech}^{2}\left[\gamma\left(J+\frac{1}{2}\right)\right]\right. \\
& \left.-\frac{\gamma}{4} \operatorname{cossech}^{2}\left(\frac{\gamma}{2}\right)\right\}
\end{aligned}
$$

O gráfico do calor específico em função de $k_{B} T / \mu_{B} H$ para um paramagneto de spin $1 / 2$, derivado a partir da equação (34), é apresentado na Figura 1.

Picos no calor específico podem ser usados como um indicador de que alguma coisa interessante está acontecendo. Neste caso, ele tem um valor máximo próximo a $k_{B} T \approx \mu_{B} H$, o qual é conhecido como anomalia de Schottky. Essa anomalia surge porque nesta temperatura é possível excitar termicamente transições entre

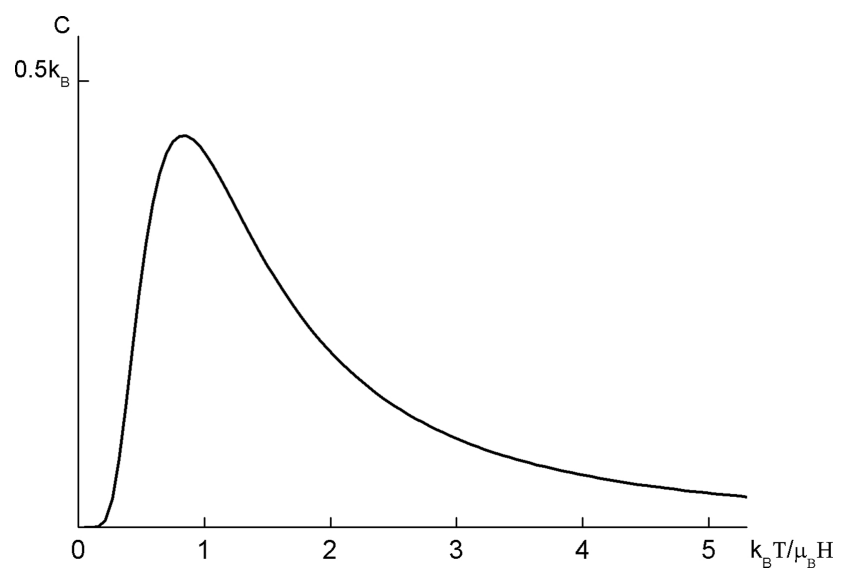

Figura 1: Calor específico em função de $k_{B} T / \mu_{B} H$ para um paramagneto de spin $1 / 2$. dois estados do sistema. Em muito baixa temperatura é difícil mudar a energia do sistema porque não existe energia suficiente para excitar transições do estado fundamental e, portanto, todos os spins permanecem presos, todos alinhados com o campo aplicado. Em temperaturas muito altas é difícil mudar a energia do sistema porque ambos os estados estão igualmente ocupados. A entropia do sistema aumenta com a temperatura, como esperado, uma vez que ela reflete a desordem dos spins, e diminui ao resfriarmos o material, correspondendo ao consequente ordenamento dos spins. Esse fato é muito usado em técnicas de refrigeração magnética 4].

\section{- Paramagnetismo de Pauli}

É a contribuição paramagnética dos elétrons de condução em um metal. Estes elétrons possuem energia dada por $\epsilon_{F}=k_{B} T_{F}$, conhecida como energia de Fermi, onde $T_{F}$ é a temperatura de Fermi. Em muitos metais esses elétrons não interagem entre si e, portanto, são descritos como um gás de elétrons, em analogia a um sistema gasoso. Para valores típicos de densidade eletrônica, $T_{F} \approx 10^{4} \mathrm{~K}$, porém, essa não é a temperatura termodinâmica dos elétrons, mas apenas um parâmetro que exerce um papel importante com relação às propriedades térmicas do gás de elétrons 12,13.

$\mathrm{Na}$ aproximação do elétron independente e considerando somente a contribuição de seu momento magnético de spin, cada elétron contribuirá com $-\mu_{B} / V$ para a densidade de magnetização se o spin está paralelo ao campo $H$ e $\mu_{B} / V$ se antiparalelo 13 . Então, se $\mathrm{n}_{ \pm}$é o número de elétrons por unidade de volume com spin paralelo $(+)$ e antiparalelo (-) a $H$, a densidade de magnetização será

$$
M=-\mu_{B}\left(n_{+}-n_{-}\right)
$$

Como os elétrons interagem com o campo $H$ através de seus momentos magnéticos, então o efeito do campo será deslocar cada nível eletrônico de $\pm \mu_{B} H$. Sendo $g(\epsilon)$ a densidade de estados em uma dada energia $\epsilon$, então $g_{ \pm}(\epsilon) d \epsilon$ é o número de elétrons com um dado spin entre $\epsilon$ e $\epsilon+d \epsilon$. Na ausência de campo teríamos

$$
g_{ \pm}(\epsilon)=\frac{1}{2} g(\epsilon) \quad(H=0)
$$

Na presença do campo cada nível é deslocado de $\pm \mu_{B} H$, então devemos ter

$$
\begin{aligned}
& g_{+}(\epsilon)=\frac{1}{2} g\left(\epsilon-\mu_{B} H\right) \\
& g_{-}(\epsilon)=\frac{1}{2} g\left(\epsilon+\mu_{B} H\right)
\end{aligned}
$$

O número total de spins por unidade de volume para cada orientação de spin é

$$
n_{ \pm}=\int d \epsilon g_{ \pm}(\epsilon) f(\epsilon)
$$


onde $f(\epsilon)$ é a função de Fermi 12,13

$$
f(\epsilon)=\frac{1}{e^{(\epsilon-\mu) / k_{B} T}+1}
$$

Em metais, os elétrons de condução estão próximos ao nível de Fermi, logo as variações importantes na densidade de estados são da ordem de $\epsilon_{F}$ e como $\mu_{B} H<<\epsilon_{F}$ para campos normais $\left(H \sim 10^{4} G\right)$, podemos expandir $g_{ \pm}(\epsilon)$ em série de Taylor e considerar os termos até primeira ordem, com erro desprezível, tal que

$$
g_{ \pm}(\epsilon)=\frac{1}{2} g\left(\epsilon \mp \mu_{B} H\right) \approx \frac{1}{2} g(\epsilon) \mp \frac{1}{2} \mu_{B} H g^{\prime}(\epsilon)
$$

Substituindo esse valor em $n_{ \pm}$, temos

$$
n_{ \pm}=\frac{1}{2} \int g(\epsilon) f(\epsilon) d \epsilon \mp \frac{1}{2} \mu_{B} H \int g^{\prime}(\epsilon) f(\epsilon) d \epsilon
$$

Portanto, a densidade de magnetização fica

$$
M=\mu_{B}^{2} H \int g^{\prime}(\epsilon) f(\epsilon) d \epsilon
$$

a qual podemos integrar por partes, para obtermos

$$
M=\mu_{B}^{2} H \int g(\epsilon)\left(-\frac{\partial f}{\partial \epsilon}\right) d \epsilon
$$

No zero absoluto, $-\partial f / \partial \epsilon=\delta\left(\epsilon-\epsilon_{F}\right)$, assim

$$
M=\mu_{B}^{2} H g\left(\epsilon_{F}\right)
$$

$\mathrm{e}$

$$
\chi=\mu_{B}^{2} g\left(\epsilon_{F}\right)
$$

No caso de elétrons livres, a densidade de estados é dada por $g\left(\epsilon_{F}\right)=3 /\left(2 \epsilon_{F}\right)$, e com isso a susceptibilidade por spin fica

$$
\chi=\frac{3 \mu_{B}^{2}}{2 k_{B} T_{F}}
$$

Como os elétrons de condução têm momentos magnéti$\cos$ de $1 \mu_{B}$, seria de se esperar uma contribuição paramagnética idêntica ao caso de Curie. No entanto, sua susceptibilidade é independente da temperatura. Isso ocorre porque os únicos elétrons capazes de inverter seu spin e, portanto, contribuirem para a susceptibilidade, são aqueles dentro de uma pequena faixa de energia de largura $k_{B} T$ em torno do nível de Fermi. Os orbitais do mar de Fermi com spins alinhados paralelamente ao campo já estão ocupados e, portanto, apenas uma fração $\mathrm{T} / T_{F}$ do número total de elétrons contribui para a susceptibilidade.

\subsection{Diamagnetismo}

O diamagnetismo está relacionado à contribuição magnética associada ao movimento orbital dos elétrons em torno do seu núcleo e, portanto, está intimamente ligado à tendência natural de oposição à penetração do campo magnético em um circuito fechado [4,5, 12, 13]. Essa resposta magnética é apresentada por alguns sólidos simples compostos por íons com todas as camadas eletrônicas preenchidas (como no caso dos halogenetos alcalinos) e também pelos elementos da família dos gases nobres, onde os momentos angulares orbital e de spin são nulos no estado fundamental [13].

Na discussão acima sobre o magnetismo dos elétrons de condução, foi exposto somente a contribuição paramagnética desses elétrons, que surge da interação entre seu spin e o campo aplicado. No entanto, contribuições diamagnéticas também estão presentes nesse caso e estão associadas ao movimento orbital dos elétrons induzido pelo campo 13]. Essa contribuição é conhecida como diamagnetismo de Landau e pode ser relacionada à contribuição paramagnética de Pauli da seguinte forma

$$
\chi_{\text {Landau }}=-\frac{1}{3} \chi_{P M, P a u l i}
$$

Aqui, nos limitaremos a um tratamento clássico do diamagnetismo, o qual consideramos satisfatório para os propósitos desse artigo.

Voltando ao caso do átomo de Hidrogênio, podemos imaginar um pequeno circuito de corrente formado por um elétron girando em torno de um núcleo positivo onde qualquer variação de fluxo magnético através desse circuito causará uma alteração no movimento orbital do elétron de tal maneira a compensar a variação de fluxo devido ao campo aplicado 12. Em outras palavras, o diamagnetismo resulta de uma perturbação do movimento orbital dos elétrons causada pela força que cada elétron experimenta quando move-se com velocidade $\vec{v}$ em um campo magnético $\vec{H}[23]$, dada por

$$
\vec{F}=e \vec{v} \times \vec{H}
$$

Supondo que $\vec{H}$ seja aplicado na direção perpendicular ao movimento do elétron, a força $\vec{F}$ será centrípeta e, com isso, causará uma pequena variação na velocidade angular do elétron $\omega$. Dessa forma, temos

$$
\begin{gathered}
e v H=\Delta\left(m_{e} \omega^{2} r\right)=2 m_{e} r \omega \Delta \omega \\
\Delta \omega=\frac{e H}{2 m_{e}}
\end{gathered}
$$

Essa variação de frequência está diretamente relacionada à variação no momento magnético orbital do elétron, que pode ser descrita como

$$
\Delta \mu=\Delta I A=\left(\frac{-Z e}{\Delta T}\right) \pi\left\langle\rho^{2}\right\rangle=\frac{-Z e \pi}{2 \pi / \Delta \omega}\left\langle\rho^{2}\right\rangle
$$

ou

$$
\Delta \mu=-\frac{Z e \Delta \omega}{2}\left\langle\rho^{2}\right\rangle
$$

onde $\left\langle\rho^{2}\right\rangle=\left\langle x^{2}\right\rangle+\left\langle y^{2}\right\rangle$ é o valor médio do quadrado da distância perpendicular entre os elétrons e as linhas de $\vec{H}$ e $\left\langle r^{2}\right\rangle=\left\langle x^{2}\right\rangle+\left\langle y^{2}\right\rangle+\left\langle z^{2}\right\rangle$ o valor médio do 
quadrado da distância entre os elétrons e o núcleo. Para uma distribuição esférica de cargas $\left\langle x^{2}\right\rangle=\left\langle y^{2}\right\rangle=\left\langle z^{2}\right\rangle$ e, portanto, $\left\langle r^{2}\right\rangle=3 / 2\left\langle\rho^{2}\right\rangle$, tal que

$$
\Delta \mu=-\frac{Z e^{2} H}{4 m_{e}}\left\langle\rho^{2}\right\rangle=-\frac{Z e^{2} H}{6 m_{e}}\left\langle r^{2}\right\rangle
$$

A susceptibilidade diamagnética fica

$$
\begin{gathered}
\chi=\frac{M}{H}=\frac{N \mu_{0} \Delta \mu}{H} \\
\chi=-\frac{N \mu_{0} Z e^{2}}{6 m_{e}}\left\langle r^{2}\right\rangle
\end{gathered}
$$

Esse é o resultado clássico de Langevin 4, 12, porém, não muito diferente do resultado obtido por cálculos de mecânica quântica para a susceptibilidade diamagnética de Larmor [13]. O resultado negativo sugere uma resposta dos momentos magnéticos contrária ao campo aplicado. Esta contribuição é desprezível se comparada à contribuição paramagnética de Curie $\left(\chi_{\text {diam }} \sim 10^{-5} \chi_{P M, \text { Curie }}\right)$, fato este que a torna perceptível somente em materiais com camada fechada, onde todos os spins estão emparelhados.

Devemos ter em mente que na abordagem da mecânica estatística clássica a ocorrência do fenômeno do diamagnetismo não é possível, pois a função de partição do sistema não depende do campo magnético externo. Dessa forma, o sistema precisa ser estudado quanticamente, levando-se em conta a interação entre o campo magnético externo e o movimento orbital dos elétrons, de tal forma que a quantização das órbitas dos elétrons produzirá a resposta diamagnética de Landau [21]. Portanto, apesar de ser possível uma análise clássica, percebemos que o diamagnetismo é um fenômeno puramente quântico.

A Figura 2 apresenta o gráfico da susceptibilidade magnética em função da temperatura para os casos do paramagnetismo de Curie e sua inversa, o paramagnetismo de

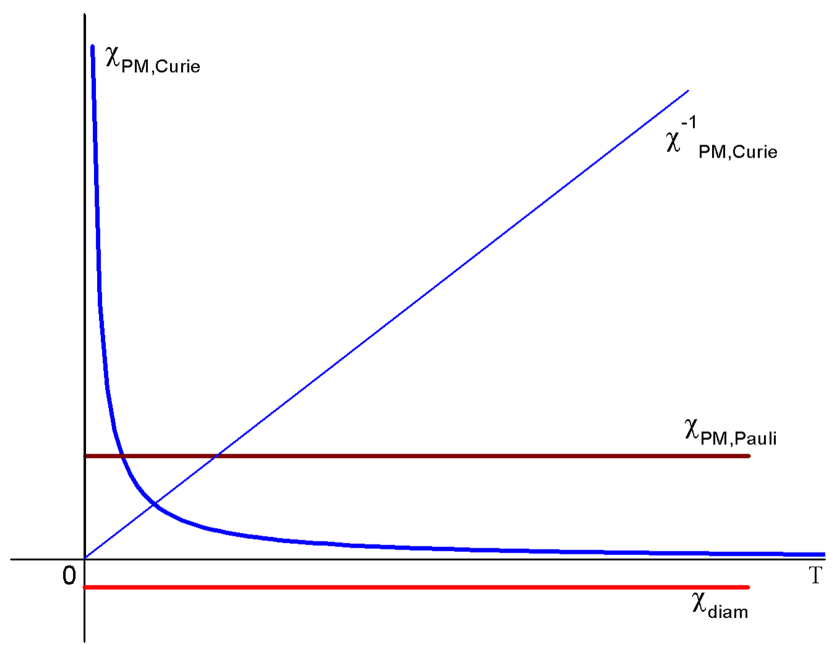

Figura 2: Susceptibilidade magnética em função da temperatura para materiais paramagnéticos (Curie e Pauli) e para materiais diamagnéticos. A inversa da susceptibilidade de um paramagneto de Curie também é apresentada.
Pauli e o diamagnetismo. Aqui, é possível perceber claramente o comportamento magnético desses materiais, com destaque para o caso paramagnético de Curie, onde as interações spin-spin localizadas aumentam drasticamente a susceptibilidade magnética em baixas temperaturas. Analisando a inversa de $\chi_{P M, C u r i e}$ vemos que ela tende a zero em $T=0$, sendo, portanto, uma identidade desse tipo de ordenamento.

Para os casos das susceptibilidades dos elétrons de condução (paramagnetismo de Pauli) e do movimento orbital eletrônico (diamagnetismo), ambas são independentes da temperatura, com valores positivos e negativos, respectivamente. Uma vez que o diamagnetismo está associado com a tendência das cargas externas em blindarem parcialmente o interior do corpo contra a ação de um campo magnético externo, seu valor negativo reflete esse fato e está em perfeito acordo com a lei de Lenz, a qual afirma que correntes induzidas se opõem à variação de campo magnético. Esse campo magnético associado com a corrente induzida cria um momento diamagnético 12.

\subsection{Ferromagnetismo}

As substâncias ferromagnéticas possuem um momento magnético espontâneo, isto é, um momento magnético que existe mesmo na ausência de campo magnético aplicado [4,5]. Esse fato pode ser compreendido se imaginarmos uma interação entre os spins cuja tendência natural seja alinhá-los em uma direção específica, como mostrado na Figura 3.

Trataremos essa interação na aproximação de Campo Médio, ou seja, imaginaremos a interação entre os spins como sendo a responsável pela geração de um campo interno $\vec{H}_{\text {int }}$ dentro do material, o qual será proporcional à própria magnetização 12 . Esse campo interno, também conhecido como campo molecular de Weiss, é da ordem de $H_{\text {int }} \approx 10^{3} \mathrm{~T}$ (para o caso do ferro), no entanto, o campo criado por um dipolo magnético, da ordem do magnéton de Bohr, em um ponto situado a uma distância de 1,0 (um) parâmetro de rede em um sólido, é da ordem de $H_{d} \approx 10^{-1} T$. Vemos, portanto, que $H_{d}<<H_{\text {int }}$ o que significa que em um ferromagneto as interações entre os dipolos magnéticos não podem ser de origem magnética.

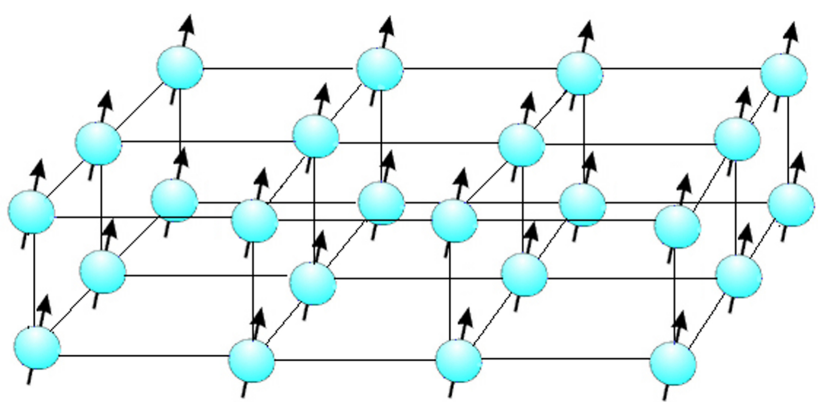

Figura 3: Ordenamento ferromagnético mostrando o ordenamento paralelo entre os spins. 
A explicação a esse fato reside na mecânica quântica com base no princípio da exclusão de Pauli, através da interação de troca (ou "exchange"), uma interação não magnética entre átomos magnéticos [24]. Então, na aproximação de Campo Médio, temos

$$
\vec{H}_{i n t}=\lambda \vec{M}
$$

onde $\lambda$ é um parâmetro, chamado de parâmetro de campo molecular.

Quando esses materiais são submetidos a um campo magnético externo, $\vec{H}$, o campo efetivo sentido por um spin localizado num sítio qualquer da rede será a soma do campo aplicado mais o campo interno [18, ou seja

$$
H_{\text {eff }}=H+H_{\text {int }}=H+\lambda M(T, H)
$$

Com o intuito de calcular o valor do parâmetro $\lambda$, escreveremos o hamiltoniano para um único spin da rede, $\vec{S}_{i}$, devido aos seus $q$ vizinhos, da seguinte forma:

$$
\mathcal{H}_{1}=-2 J_{i j} \vec{S}_{i} \cdot \sum_{j=1}^{q} \vec{S}_{j}
$$

onde $J_{i j}$ é o parâmetro de troca entre os spins $\vec{S}_{i}$ e $\vec{S}_{j}$. Podemos ainda reescrever a equação (58) como

$$
\mathcal{H}_{1}=-g \mu_{B}\left(\frac{2 J_{i j}}{g \mu_{B}} \sum_{j=1}^{q} \vec{S}_{j}\right) \cdot \vec{S}_{i}
$$

Dessa forma, podemos identificar a quantidade entre parênteses como sendo o campo produzido pela soma dos $q$ spins $\vec{S}_{j}$ na posição do spin $\vec{S}_{i}$, ou seja, é exatamente o campo sentido pelo spin $\vec{S}_{i}$ devido às interações dos seus vizinhos da rede, o qual havíamos definido anteriormente. Assim, ficamos com

$$
\mathcal{H}_{1}=-g \mu_{B} \vec{H}_{i n t} \cdot \vec{S}_{i}
$$

Como estamos usando a aproximação de campo médio, podemos substituir $\vec{S}_{j}$ por $<S_{j}>$

$$
H_{i n t}=\frac{2 q J_{i j}}{g \mu_{B}}<S_{j}>
$$

Como $<S_{j}>$ está diretamente relacionado à magnetização do material, $M=N g \mu_{B}<S_{j}>$, podemos escrever o campo magnético de interação, sentido pelo spin $\vec{S}_{i}$, como

$$
H_{i n t}=\frac{2 q J_{i j}}{g \mu_{B}}\left(\frac{M}{N g \mu_{B}}\right)=\frac{2 q J_{i j}}{N g^{2} \mu_{B}^{2}} M
$$

Portanto, de acordo com a equação (56), o parâmetro de campo molecular será

$$
\lambda=\frac{2 q J_{i j}}{N g^{2} \mu_{B}^{2}}
$$

Com a determinação do parâmetro de campo molecular e, consequentemente, do campo de interação efetivo, podemos proceder de maneira análoga ao tratamento realizado anteriormente, ou seja, podemos imaginar a equação (11) como sendo o hamiltoniano de um spin na presença de um campo efetivo (equação 57) e escrever uma expressão análoga à equação (24) para a magnetização na presença desse campo da seguinte forma:

$$
M=N g \mu_{B} S B_{S}(x)=N g \mu_{B} S B_{S}\left(\frac{g \mu_{B} S H_{e f f}}{k_{B} T}\right)
$$

Aqui, o campo que entra no argumento da função de Brillouin é o campo efetivo $H_{\text {eff }}$ sentido pelo spin $\vec{S}_{i}$.

No regime de altas temperaturas, onde a energia térmica é muito maior que a energia magnética $\left(k_{B} T>>\right.$ $g \mu_{B} H$ ou $\left.x<<1\right)$, podemos expandir $B_{S}(x)$ em série de Taylor e tomarmos somente os termos até primeira ordem, analogamente ao caso paramagnético

$$
\begin{gathered}
M=\frac{N g^{2} \mu^{2} S(S+1)}{3 k_{B} T} H_{e f f}=\frac{C H_{e f f}}{T} \\
M=\frac{C}{T}(H+\lambda M)
\end{gathered}
$$

$\mathrm{ou}$

$$
M=\frac{C H}{T-\lambda C}
$$

onde $C=N g^{2} \mu^{2} S(S+1) / 3 k_{B}$ é a constante de Curie para o caso ferromagnético.

O parâmetro $\lambda C=\theta_{C W}$ é conhecido como temperatura de Curie-Weiss. Com isso, podemos escrever a susceptibilidade magnética de um ferromagneto como

$$
\chi=\frac{C}{T-\theta_{C W}}
$$

A temperatura de Curie-Weiss determina o ponto onde os momentos magnéticos perdem sua magnetização espontânea, portanto, ela separa a fase ordenada ferromagnética $\left(T<\theta_{C W}\right)$ da fase paramagnética $\left(T>\theta_{C W}\right)$. Por esse motivo, ela é também chamada de temperautra de transição ferromagnética. A constante de Curie é particularmente importante, pois ela fornece, dentre outras coisas, uma estimativa da concentração dos íons do material.

Em temperaturas muito menores do que a temperatura de Curie-Weiss, os momentos magnéticos de uma substância ferromagnética estão praticamente paralelos em escala microscópica. Entretanto, para a amostra como um todo, o momento magnético pode ser muito menor do que o momento de saturação. Esse comportamento é atribuído ao que chamamos de domínios magnéticos (região do material dentro da qual todos os átomos têm o mesmo alinhamento magnético) 12 13. Um material ferromanético, em geral, é caracterizado por vários domínios magnéticos, onde os momentos podem alinhar-se em uma direção específica dentro desse domínio, porém, podem 
variar drasticamente sua direção entre domínios diferentes, resultando em uma magnetização completamente diferente de seu valor teórico de saturação.

Os domínios são responsáveis, por exemplo, pelo ciclo de histerese apresentado por certos materiais ferromagnéticos 4, 5, 12,13. Nele, a magnetização inicialmente nula (em campo nulo) aumenta continuamente até seu valor de saturação ao aumentarmos o campo aplicado. Se invertermos a direção de aplicação do campo, cada spin (dentro de seu domínio) estará sujeito a um torque cuja ação tende a inverter sua orientação, no entanto, as paredes de domínio exercem forças coercivas dificultando essa inversão, resultando em uma magnetização diferente de zero a campo nulo (magnetização remanente). O resultado é uma curva $\vec{M}(\mathrm{H})$ como mostrado na Figura 4 . O valor do campo onde a magnetização torna-se nula é chamado de campo coercivo, $H_{c}$. É através deste campo que definem-se os materiais magneticamente macios (baixos valores de $H_{c}$ ) e os magneticamente duros (altos valores de $H_{c}$ ).

\subsection{Antiferromagnetismo}

Alguns materiais exibem um comportamento magnético completamente diferente daqueles estudados até aqui, como é o caso do composto intermetálico $\mathrm{YbRh}_{2} \mathrm{Si}_{2}$ [25]. Essa classe de materiais é caracterizada pela não existência de um momento magnético total líquido (em baixas temperaturas) na ausência de um campo externo, mesmo apresentando fortes interações entre seus spins. Ao baixarmos a temperatura continuamente, sua susceptibilidade magnética atinge um valor máximo e depois volta a cair, chegando a anular-se em baixíssimas temperaturas $4,5,12,13$. Isso ocorre devido à existência de fortes interações entre seus spins localizados que dão origem a uma fase ordenada abaixo de um valor característico de temperatura, conhecido como temperatura de Néel $\left(T_{N}\right)$. Esse fenômeno, até então observado somente por medidas indiretas (como anomalias no calor específico),

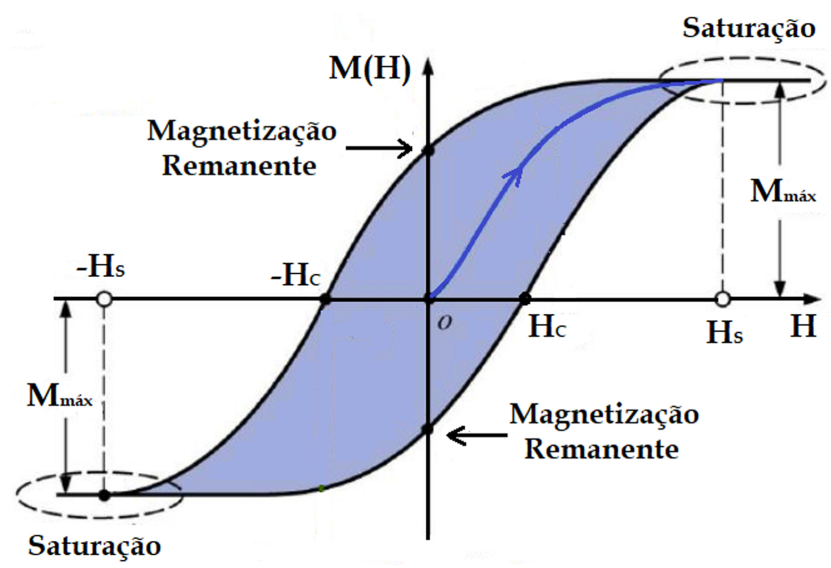

Figura 4: Curva de histerese típica de materiais ferromagnéticos. Nela é possível observar a variação da magnetização em função do campo aplicado. foi confirmado experimentalmente por C.G. Shull e J.S. Smart em 1949 com a utilização da difração de nêutrons em $\mathrm{MnO}$ [26]. Tal técnica fornece uma maneira direta de detecção do antiferromagnetismo, pois a interação dos nêutrons magnéticos com a rede resulta em um espalhamento coerente mensurável. A existência desse padrão espacial ordenado dos momentos magnéticos individuais favorece uma disposição antiparalela aos seus vizinhos próximos. Podemos, então, pensar nesse estado como sendo composto por spins formando duas sub-redes, A e B, cada uma delas ordenadas ferromagneticamente e opostas entre si, resultando em uma magnetização nula no zero absoluto (ver Figura 5). Vistas assim, as subredes A e B estarão dispostas de tal maneira que os primeiros vizinhos de um íon da sub-rede A estão todos contidos na sub-rede B e vice-versa.

Usaremos a aproximação de Campo Médio, separadamente para as sub-redes A e B, de tal forma que um íon localizado na sub-rede A sentirá um campo efetivo $H_{B}$ e um íon localizado na sub-rede B sentirá um campo efetivo $H_{A}$, dados por

$$
\begin{aligned}
& H_{A}=-\Lambda M_{B}-\Gamma M_{A} \\
& H_{B}=-\Lambda M_{A}-\Gamma M_{B}
\end{aligned}
$$

Aqui, $M_{A}$ e $M_{B}$ são as magnetizações das sub-redes A e $\mathrm{B}$, e $\Lambda$ e $\Gamma$ são os parâmetros de troca com os primeiros e segundos vizinhos, respectivamente. O parâmetro $\Lambda$ favorece, portanto, um alinhamento antiparalelo entre os spins $(\Lambda<0)$ e $\Gamma$ favorece um alinhamento paralelo $(\Gamma>$ $0)$. Devido às sub-redes serem exatamente equivalentes na ausência de campo aplicado, podemos afirmar que $M_{A}$ será igual, em magnitude, a $M_{B}$.

Olhando para cada sub-rede individualmente, vemos exatamente a mesma situação apresentada anteriormente, portanto, as expressões para suas magnetizações serão análogas àquela dada pela equação (64), ou seja,

$$
M=\frac{1}{2} N g \mu_{B} S B_{S}\left(\frac{g \mu_{B} S H_{e f f}}{k_{B} T}\right)
$$

onde o fator $1 / 2$ refere-se à contribuição de cada sub-rede para a magnetização total.

Para derivar o comportamento da susceptibilidade de um antiferromagneto acima da temperatura crítica $(T>$

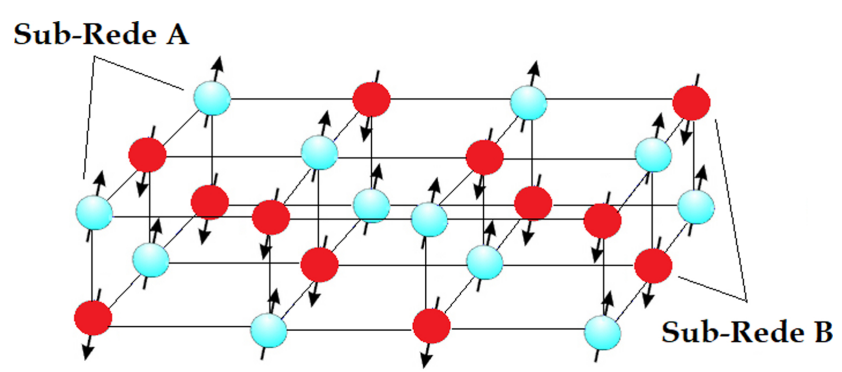

Figura 5: Ordenamento antiferromagnético mostrando as duas sub-redes $A$ e $B$ com seus spins dispostos antiparalelamente. 
$T_{N}$ ), notemos que tanto $\vec{M}_{A}$ quanto $\vec{M}_{B}$ são pequenas e somente serão induzidas na presença de um campo magnético aplicado. Assim, o comportamento da função de Brillouin será idêntico ao caso ferromagnético

$$
B_{S}(x) \rightarrow \frac{(S+1) x}{3 S} \quad \text { para } \quad x \rightarrow 0
$$

As magnetizações das duas sub-redes tenderão a se alinhar com o campo aplicado e, dessa forma, os parâmetros $\Lambda$ e $\Gamma$ terão o mesmo sinal. Assim, as expressões para $M_{A}$ e $M_{B}$ ficam

$$
M_{A}=\frac{1}{2} N g \mu_{B} S\left(\frac{S+1}{3 S}\right) \frac{g \mu_{B} S}{k_{B} T}\left[-(\Lambda+\Gamma) M_{A}+H\right]
$$

e

$$
M_{B}=\frac{1}{2} N g \mu_{B} S\left(\frac{S+1}{3 S}\right) \frac{g \mu_{B} S}{k_{B} T}\left[-(\Lambda+\Gamma) M_{B}+H\right]
$$

Para encontrarmos a susceptibilidade magnética, precisamos levar em consideração as duas magnetizações, ou seja

$$
\chi=\frac{M_{A}+M_{B}}{H}
$$

que podemos encontrar facilmente com o auxílio das equações 72 e 73 )

$$
\chi=\frac{N g^{2} \mu_{B}^{2} S(S+1) / 3 k_{B}}{T+\frac{1}{2}\left[N g^{2} \mu_{B}^{2} S(S+1) / 3 k_{B}\right](\Lambda+\Gamma)}
$$

Identificando, para este caso, a constante de Curie-Weiss como sendo $\theta_{C W}=\frac{1}{2} C(\Lambda+\Gamma)$, a susceptibilidade magnética para um material antiferromagnético fica

$$
\chi=\frac{C}{T+\theta_{C W}}
$$

Na presença de um ordenamento antiferromagnético, o campo interno gerado pelos spins do material apontará na direção oposta ao campo aplicado externamente.

A Figura 6 mostra o comportamento da susceptibilidade magnética em função da temperatura e também sua inversa no regime $k_{B} T>>g \mu_{B} H$ para os ordenamentos ferromagnético e antiferromagnético. No caso de um antiferromagneto, a suscetibilidade é finita em $T=T_{N}$, apresentando nesse limite um vértice (ou pico) pouco acentuado na curva do plano $\chi-T$. É importante mencionar também que, uma transição similar no limite $T \rightarrow T_{N}$ também é observada para a capacidade calorífica e o coeficiente de expansão térmica. Existe também uma impressão magnética crítica para o caso particular do calor específico em campo nulo em função da temperatura, onde são observadas singularidades características difíceis de lidar teoricamente 12,13.

A curva $\chi(T)$ de um FM diverge diretamente em $\theta_{C W}$, enquanto que a curva de um AFM cai a zero abaixo de $T_{N}$, no entanto, seu prolongamento (linha tracejada) nos mostra que ela teria um comportamento divergente no

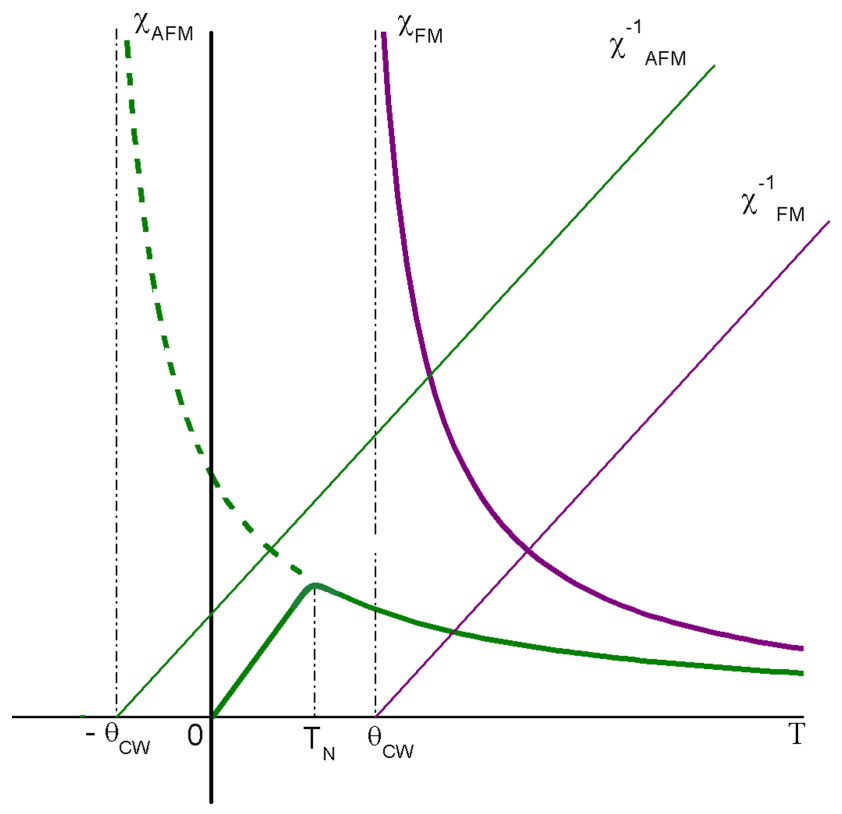

Figura 6: Susceptibilidade magnética e sua inversa em função da temperatura para materiais ferromagnéticos e antiferromagnéticos.

ponto $-\theta_{C W}$, o que é evidenciado pela sua inversa. $\mathrm{Na}$ prática, para um material AFM, os valores de $-\theta_{C W}$ e $T_{N}$ em geral não são iguais (em módulo), contrariando a equação (76). Isso se deve às interações entre os vizinhos mais próximos e às diferenças nos arranjos das sub-redes 12.

Existem outros tipos de acoplamentos mais complexos que surgem em baixas temperaturas, como o ferrimagnetismo, o qual apresenta duas sub-redes opostas, porém, não equivalentes e, portanto, não se cancelam, resultando em uma componente magnética em uma dada direção. Existe também um tipo de ordenamento conhecido como helimagnetismo, onde os átomos magnéticos são dispostos em camadas de tal forma que dois spins localizados em camadas consecutivas formam um ângulo $\theta$ entre si. Um outro tipo de arranjo ordenado, porém, menos comum, é o chamado "spin glass", composto por um sistema de spins aleatórios com múltiplas interações, porém, ainda assim caracterizado por uma "temperatura de congelamento" bem definida, abaixo da qual um estado metaestável aparece sem o usual ordenamento de longo alcance [4,5]. Apesar de presentes em uma vasta gama de materiais como as ferritas, ligas metálicas contendo elementos de terras raras e ligas não magnéticas dopadas com átomos magnéticos, respectivamente, esses tipos de ordenamentos não serão abordados aqui.

\section{Conclusão}

Neste trabalho estudamos as propriedades magnéticas da matéria através de uma abordagem estatística, analisando suas interações microscópicas de modo a descrever seu comportamento em função da temperatura. Aqui, investi- 
gamos diferentes classes de materiais e suas principais características, do ponto de vista da teoria clássica, mesmo que em determinados aspectos a Mecânica Quântica tenha sido acionada por ser o tratamento mais adequado. Os procedimentos descritos no texto visam esclarecer, de forma simplificada, todos os aspectos referentes às principais propriedades magnéticas dos materiais e, dessa forma, auxiliar estudantes e leitores no entendimento do magnetismo.

\section{Referências}

[1] I.R.O. Ramos, J.P.M. Braga, J.W.C. Silva, A.P. Lima e L.M. Holanda, Revista Brasileira de Ensino de Física 39, e2306 (2017).

[2] I.R.O. Ramos, J.P.M. Braga, J.V.A. Ataíde e A.P. Lima, Revista Brasileira de Ensino de Física 40, e5408 (2018).

[3] G.A.P. Ribeiro, Revista Brasileira de Ensino de Física 22, 299 (2000).

[4] S. Blundell, Magnetism in Condensed Matter (Oxford University Press, New York, 2001).

[5] K.H.J. Buschow e F.R. de Boer, Physics of Magnetism and Magnetic Materials (Kluwer Academic/Plenum $\mathrm{Pu}-$ blishers, New York, 2003).

[6] P. Pesic, Labyrinth: A Search for the Hidden Meaning of Science (The MIT Press, Cambridge, 2000).

[7] D.J. Griffiths, Introduction to Electrodynamics (Prentice Hall, New Jersey, 1999).

[8] J.D. Jackson, Classical Electrodynamics (John Wiley \& Sons, New Jersey, 1999).

[9] H.B. Lal e V. Pratap, Journal of Materials Science 17, 377 (1982).

[10] J. Jensen e A.R. Mackintosh, Rare Earth Magnetism: Structures and Excitations (Clarendon Press, Oxford, 1991).

[11] B.G. Wybourne, Spectroscopic Properties of Rare Earths (John Wiley \& Sons, New York, 1965).

[12] C. Kittel, Introdução à Física do Estado Sólido (Guanabara Dois, Rio de Janeiro, 1978).

[13] N.W. Ashcroft e N.D. Mermin, Solid State Physics (Harcourt College Publishers, New York, 1976).

[14] D. Halliday, R. Resnick e J. Walker, Fundamentos de Física (LTC, Rio de Janeiro, 2012), v. 3.

[15] H.M. Nussenzveig, Curso de Física Básica (Edgard Blücher, São Paulo, 1997), v. 3.

[16] J.J. Sakurai, Modern Quantum Mechanics: Revised Edition (Addison-Wesley, Los Angeles, 1994).

[17] D.J. Griffiths, Introduction to Quantum Mechanics (Prentice Hall, New Jersey, 1995).

[18] J.S. Smart, Effective Field Theories of Magnetism (Saunders, Philadelphia \& London, 1966).

[19] H.B. Callen, Thermodynamics and an Introduction to Thermostatistics (John Wiley \& Sons, Singapore, 1985).

[20] W. Greiner, L. Neise e H. Stöcker, Thermodynamics and Statistical Mechanics (Springer, New York, 1997).

[21] S.R.A. Salinas, Introdução à Física Estatística (EDUSP, São Paulo, 2005).

[22] M.I. Darby, British Journal of Applied Physics 18, 1415 (1967).

[23] D.H. Martin, Magnetism in Solids (M.I.T. Press, Cambridge, 1967).
[24] M.J. Oliveira, Termodinâmica (Livraria da Física, São Paulo, 2005).

[25] J. G.S. Duque, E.M. Bittar, C. Adriano, C. Giles, L.M. Holanda, R. Lora-Serrano, P.G. Pagliuso, C. Rettori, C.A. Pérez, R. Hu et al., Physical Review B 79, 035122 (2009).

[26] C. G. Shull e J. Samuel Smart, Phys. Rev. 76, 1256 (1949). 